\title{
Tunable symmetry and periodicity in binary supramolecular nanostructures
}

\author{
Dimas G. de Oteyza, ${ }^{a, b}$ Esther Barrena, ${ }^{c, d}$ Helmut Dosch, ${ }^{c, e}$ J. Enrique Ortega, ${ }^{f, g}$ Yutaka Wakayama ${ }^{b}$ \\ Received (in $X X X, X X X)$ Xth $X X X X X X X X X 200 X$, Accepted Xth XXXXXXXXX 200X \\ ${ }_{5}$ First published on the web Xth $X X X X X X X X X 200 X$ \\ DOI: 10.1039/b000000x
}

\begin{abstract}
We present a route to change the "compositional" order of highly crystalline binary layers comprising diindenoperylene and copper-phthalocyanines from two- into one-dimensional 10 periodicity. This is achieved exchanging fluorine by hydrogen atoms in the phtalocyanines, thereby reducing the C-F...H-C interactions and allowing the interplay of long-range electrostatic interactions in mesoscopic phases. Linear patterns are thus obtained, whose periodicity can be additionally tuned by an 15 appropriate stoichiometry of the components.
\end{abstract}

Molecular electronics is envisioned as one of the possible breakthroughs in electronics and information technology. Progress in the control of supramolecular self-assembly is expected to make it possible, paving the way towards tailored 20 synthesis of functional molecular nanostructures. However, the assembly of appropriate molecules into well ordered arrays on technologically relevant surfaces is a challenge that demands the control of system interactions at different length scales. At the nanoscopic level, the process is determined by a 25 complex interplay between intermolecular and moleculesubstrate interactions. Several routes have been exploited to steer those interactions. A handle on molecule-substrate interactions is provided by the use of nanostructured surfaces. This has been demonstrated with surface superstructures or 30 stepped surfaces, which can, for example, define preferential adsorption sites. ${ }^{1-5}$ In turn, the use of appropriate molecules with complementary functional groups allows the formation of strong intermolecular interactions. These include hydrogen bonds, ${ }^{5-10}$ or even chemical reactions, ${ }^{11,12}$ both of whose 35 directionality provides an additional control parameter in the design of molecular arrays.

Ideally, long-range ordered molecular arrays with tunable symmetry and periodicity are desired. A beautiful example thereof recently reported are molecular gratings made of 40 methionine stripes on silver surfaces, ${ }^{13}$ which have been used as templates for the subsequent growth of atomic strings. ${ }^{14}$ Their tunable periodicity (via methionine coverage), together with their behavior as one-dimensional electron resonators, allows tailoring the characteristics of quantum-well states. ${ }^{15}$ ${ }_{45}$ Supramolecular self-assembly of two- (or multi-) component systems has been repeatedly used for successful synthesis of highly ordered nanostructured layers with diverse crystalline structures. Most examples report the formation of well ordered two-dimensional molecular mixtures.5678, 16-31, A

50 lesser number reports the formation of crystalline structures with each of the molecular components arranged in separate rows and therefore leading to one-dimensional "compositional" order. ${ }^{28-35}$ Only few of the aforementioned examples show tunable properties of their symmetry 55 (changing e.g. between one- and two-dimensional structures) or periodicity, which is achieved either by adjusting the molecular ratio $9^{, 16,25,30,31}$ or by annealing treatments. ${ }^{26,29,32}$ In this letter, we demonstrate the formation of supramolecular nanostructures with tunable properties of both their symmetry 60 and periodicity, by means of adjustment of the intermolecular interactions and of the binary layer stoichiometry of copperphthalocyanines and diindenoperylene (DIP, Fig. 1a).

The complementary functionalities of fluorinated copperphthalocyanines $\left(\mathrm{F}_{16} \mathrm{CuPc}\right.$, Fig. 1a) and DIP give rise to rather ${ }_{65}$ strong intermolecular interactions via $\mathrm{C}-\mathrm{H} \cdots \mathrm{F}-\mathrm{C}$ bonds. ${ }^{36,37}$ As a consequence, in order to maximize them, growth of binary layers in a 1:1 ratio on $\mathrm{Au}(111)$ and $\mathrm{Cu}(111)$ surfaces leads to the formation of highly crystalline two-dimensional structures with the molecules arranged in such a way that each 70 molecule is surrounded by the opposite species (Fig. 1b). 16,36,37 However C-H $\cdots$ F-C interactions can be eliminated by exchanging $\mathrm{F}_{16} \mathrm{CuPc}$ by its homeomorphic non-fluorinated counterpart $\mathrm{CuPc}$ (Fig. 1a), whose combination with DIP in binary layers is therefore expected to present a much lower ${ }_{75} 2 \mathrm{D}$ cohesive energy.

Our results show that indeed, in DIP-CuPc binary layers, the molecules do not surround themselves by the opposite specie. In contrast, they arrange into alternating molecular rows, finally producing well ordered layers with one80 dimensional compositional periodicity (Fig. 1c) and domains of up to several tens of nanometers (Fig. S1).

Such findings apply to different substrates, demonstrated here with $\mathrm{Au}(111)$ and $\mathrm{Cu}(111)$. Figure 2 shows small scale STM images of 1:1 binary layers on both substrates. The 85 similarity among the structures obtained on either surface is evident, and the main difference is related to their symmetry.

In the case of $\mathrm{Au}(111)$ we find two different molecular orientations within the rows of each molecular specie. The two azimuthal orientations of each molecule are related by a 90 mirror plane directed along the molecular rows, evidencing their alignment with the high symmetry directions of the substrate. In particular, the rows have been found to align along the $\mathrm{Au}$ [1-10] and equivalent directions, giving rise to three discrete row orientations separated by $60^{\circ}$, each of them 95 additionally displaying two equivalent mirror domains. The frequent changes in molecular orientation associated to the two mirror domains indicate the low energy associated to such domain boundaries.

In contrast, on $\mathrm{Cu}(111) \mathrm{DIP}$ and $\mathrm{CuPc}$ molecules present a 
(a)

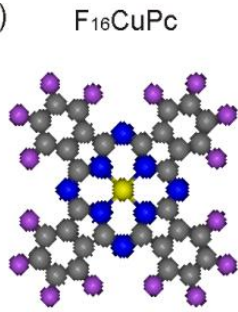

(b) $\mathrm{F}_{16} \mathrm{CuPc} \& \mathrm{DIP}$

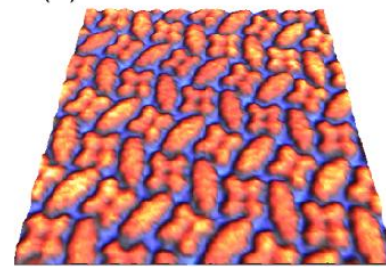

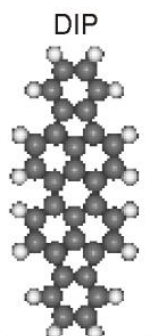

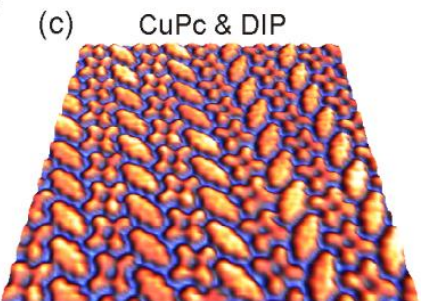

Fig. 1 (a) Schematic representation of $\mathrm{F}_{16} \mathrm{CuPc}$, DIP and $\mathrm{CuPc}$ molecules. (b) $12 \times 12 \mathrm{~nm}^{2}$ STM image of a binary layer combining DIP with $\mathrm{F}_{16} \mathrm{CuPc}$ on $\mathrm{Au}(111)$ in a 1:1 ratio. (c) The same as (b) combining DIP with CuPc.

5 single orientation for each direction of the molecular rows, and we find six different directions related by 30 degrees rotations. This is the result of the molecular rows being directed 15 degrees off the high symmetry directions (Fig. $2 b$ ), which leads to the presence of three rotational and three 10 mirror domains alternating and equidistant by 30 degrees.

The parameters of the unit cells outlined in Fig. 2, which comprise one $\mathrm{CuPc}$ and one DIP molecule, are given in Table 1 for both substrates, together with the respective molecular orientations within the cell, $\chi$ and $\phi$, which correspond to the 15 angle between vector $\boldsymbol{b}$ and the CuPc diagonal or the long DIP axis, respectively. Below the STM images in Fig. 2, we have plotted schemes of the proposed molecular arrangement in relation to the substrate surface structure. Based on the experimental input, the models show the closest matching 20 overstructure compatible with the highest possible degree of epitaxy (commensurate on $\mathrm{Au}(111)$ and coincident on $\mathrm{Cu}(111)){ }^{38}$ The structural parameters and epitaxial matrix are given in Table 1 for comparison with the experimental data. It can be seen that the proposed epitaxial structures fit 25 excellently to the experimental data in terms of parameters and domain orientations.

Based on the similarity of the overlayers on $\mathrm{Au}(111)$ and $\mathrm{Cu}(111)$, whose error margins of the unit cell parameters largely overlap, one single structure common to both substrate 30 surfaces cannot be ruled out. However, such case would decrease the degree of epitaxy with the substrates at least for one of the overlayers. A similar linear CuPc-DIP pattern has been observed on $\operatorname{Ag}(111),{ }^{39}$ but for this surface the binary mixture $\mathrm{F}_{16} \mathrm{CuPc}$-DIP remains unexplored and a change from 35 one- to two-dimensional compositional order by controlled modification of the intermolecular interactions has not yet been proved.

Such film structures with one-dimensional compositional periodicity can be interpreted as phase separation of DIP and ${ }_{40} \mathrm{CuPc}$ into periodically arranged striped domains (corresponding to the molecular rows). We suggest that, in competition with intermolecular interactions (including steric effects and van der Waals interaction), electrostatic
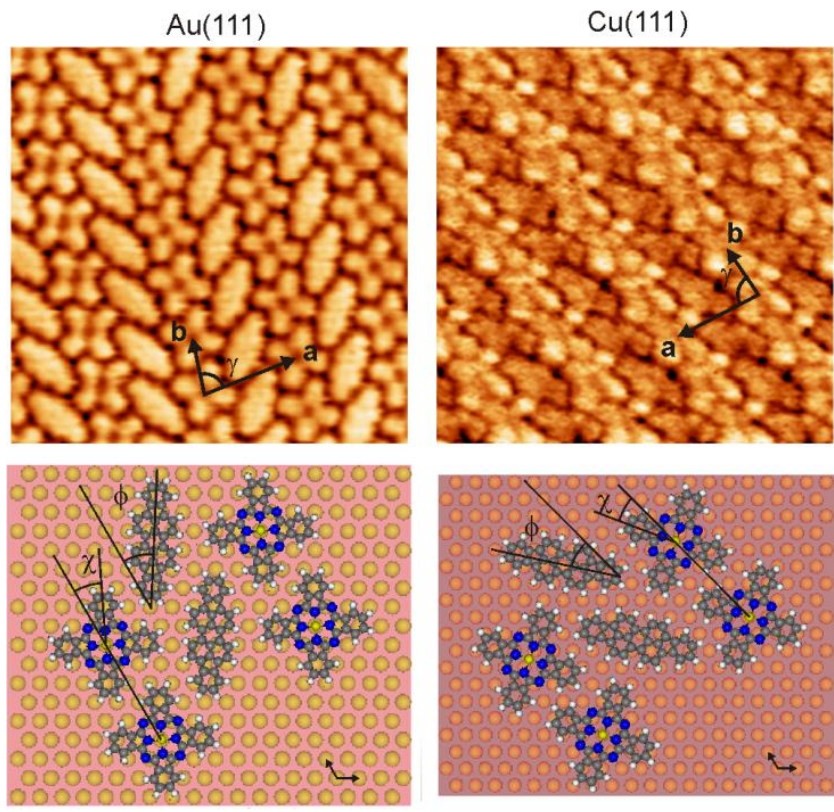

45 Fig. $210 \times 10 \mathrm{~nm}^{2}$ STM images of the CuPc:DIP 1:1 crystalline structures on $\mathrm{Au}(111)$ and $\mathrm{Cu}(111)$ surfaces (top) together with the corresponding epitaxial models (bottom). For the model on $\mathrm{Au}(111)$, the herringbone reconstruction has been neglected in spite of its experimental observation. Consequently, lattice mismatches of up to $4 \%$ may occur in dependence 50 of the reconstruction domain orientation. The inclusion of such effect into the model is hindered by the extension of the molecular domains throughout multiple rotational domains of the substrate reconstruction.

interactions may play an important role, the latter resulting either from intrinsic molecular dipoles or from absorption55 induced surface dipoles. Such surface dipoles are responsible for the commonly observed work function changes of metal surfaces upon molecular adsorption and may largely vary from molecule to molecule, being specific to each moleculesubstrate interface. ${ }^{40}$ According to previous works, energy 60 minimization by dipole-dipole interactions in binary systems (with stoichiometries in the range of those used for the present experiments) leads to segregation into striped phases. ${ }^{41,42}$

Thus, although other aspects such as epitaxial relations with the substrate may play additional important roles in the 65 resulting film structure (as discussed above), it seems plausible to ascribe a decisive role in the linear molecular arrangements of these binary layers to long-range dipoledipole interactions. In this context, the shortest possible periodicity (alternating, single molecular rows) points to very 70 low phase boundary energies allowing for maximized dipoledipole interactions.

However, most important is the fact that a controlled reduction of the intermolecular interactions simply exchanging fluorine by hydrogen atoms, yields remarkable 75 changes from two- to one-dimensional periodicities in the crystalline structures.

It is worth noting that a similar attempt was reported on binary layers combining nickel-tetraphenylporphyrin (NiTPP) with the fluorinated $\left(\mathrm{F}_{16} \mathrm{CoPc}\right)$ and non-fluorinated $(\mathrm{CoPc})$ ${ }_{80}$ cobalt-phthalocyanines. ${ }^{24}$ However, the experiment failed to find a tunability of the crystalline properties of such layers, given the disordered nature of the NiTTP-CoPc mixture. 
Table 1 Structural parameters of the 1:1 crystalline phases on $\mathrm{Au}(111)$ and $\mathrm{Cu}(111)$ from experiment and epitaxial models.

\begin{tabular}{|c|c|c|c|c|c|c|c|}
\hline \multirow{3}{*}{$\mathrm{Au}(111)$} & & $\mathrm{a}$ & b & $\gamma$ & $\chi$ & $\phi$ & \multirow[t]{3}{*}{ Matrix } \\
\hline & Exp. & $25.4 \pm 0.9$ & $14.3 \pm 0.4$ & $83 \pm 3$ & $24 \pm 4$ & $34 \pm 4$ & \\
\hline & Model & 25.1 & 14.4 & 83.4 & 26 & 33 & \\
\hline \multirow[t]{2}{*}{$\mathrm{Cu}(111)$} & Exp. & $24.4 \pm 1$ & $13.8 \pm 0.5$ & $83 \pm 3$ & $24 \pm 4$ & $33 \pm 4$ & \\
\hline & Model & 24.7 & 13.5 & 83.8 & 25 & 33 & \\
\hline
\end{tabular}

${ }^{a}$ This matrix is only approximate, as deviations arising due to the surface reconstruction are neglected.
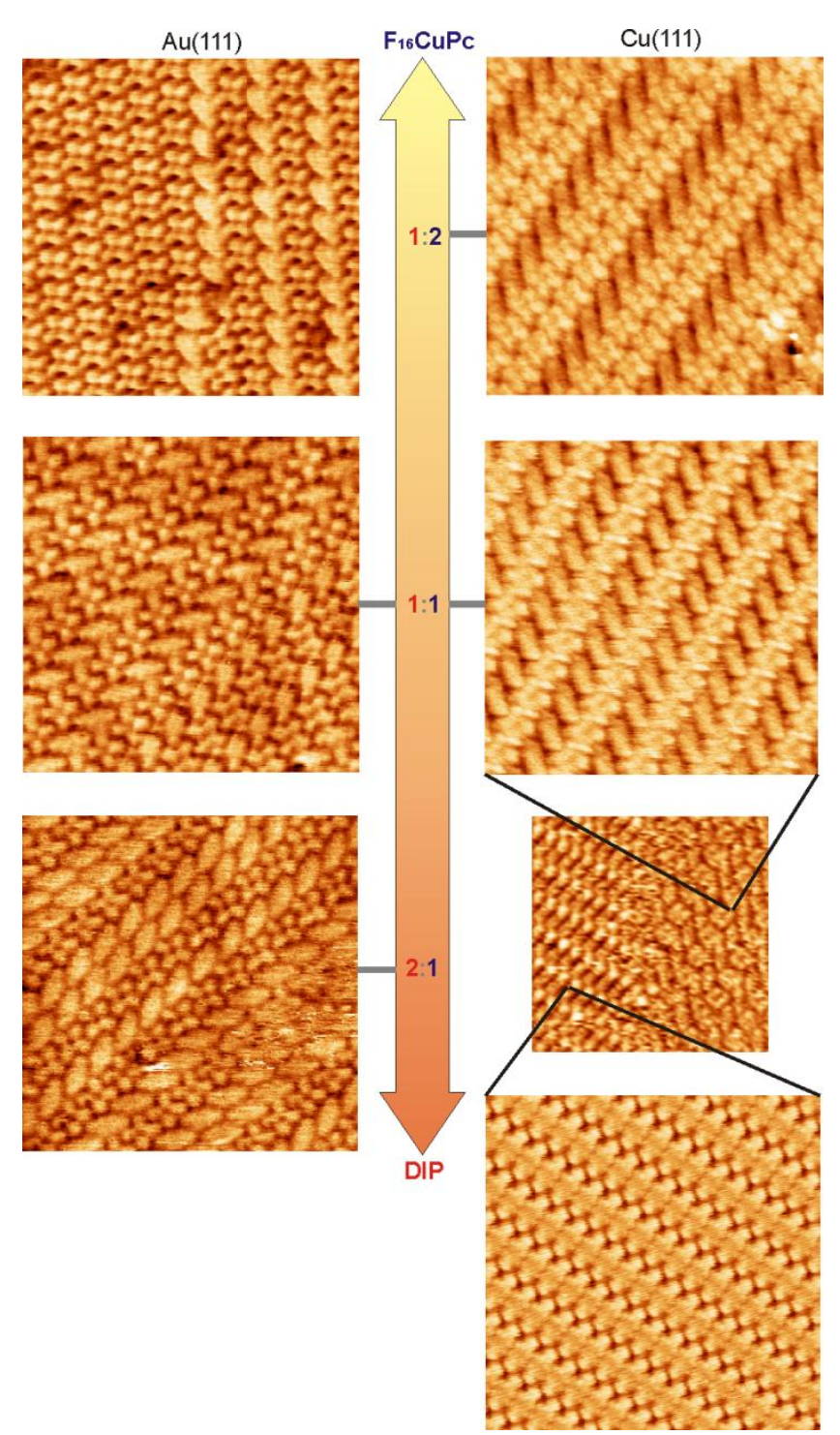

5 Fig. $314 \times 14 \mathrm{~nm}^{2}$ STM images of the resulting structures for binary layers combining DIP with $\mathrm{CuPc}$ with varying ratios on $\mathrm{Au}(111)$ and $\mathrm{Cu}(111)$ surfaces. For better resolution in the 2:1 structure image on $\mathrm{Cu}(111)$, an image of the pure DIP region is shown, and we refer to the 1:1 structure in the binary regions. Marked with grey lines are those systems leading

10 to periodic linear patterns.

The structures obtained modifying the CuPc:DIP stoichiometry on either surface are further summarized in Figure 3. Being already published previous works on single component $\mathrm{CuPc}$ or DIP on both surfaces, ${ }^{47-47}$ we only focus 15 on binary layers with molecular ratios of $2: 1$ and $1: 2$. Most interestingly, we find that the periodicity of the onedimensional structures can be tuned via the stoichiometry. For the CuPc:DIP 2:1 ratio we find the following. On $\mathrm{Au}(111)$ we either observe phase separation into pure CuPc and binary 1:1 20 regions, or a disordered mixture (alternating double, triple, or higher multiplicity $\mathrm{CuPc}$ rows with those of DIP). However, on $\mathrm{Cu}(111)$ the binary layer keeps an ordered arrangement of alternating molecular rows, now featuring double rows for the $\mathrm{CuPc}$. The result is thus a highly regular linear pattern 25 comparable to the $1: 1$ structure but with an increased periodicity.

A comparable scenario appears for the $2: 1$ ratio, though in this case it is on the $\mathrm{Cu}(111)$ where we observe phase separation into pure DIP and binary regions, both of them 30 highly ordered. ${ }^{48}$ Segregation of pure DIP beyond the $1: 1$ stoichiometry and the absence of disordered mixtures with alternating molecular rows of randomly varying multiplicity points to the DIP structure as a particularly low energy phase, which alters the mesoscopic balance of dipole-dipole and 35 phase boundary energies. In contrast, on $\mathrm{Au}(111)$ the onedimensional compositional order is maintained. Now it is the DIP which features double molecular rows alternating with those of CuPc. The presence of single DIP rows in the STM image is related to a slightly deviated stoichiometry, but the 40 absence of disorder or phase separated regions points to potentially highly periodic linear patterns upon a more precise stoichiometric deposition.

The differences in the 1D ordering for different stoichiometries of the CuPc:Dip mixtures on $\mathrm{Au}(111)$ and ${ }_{45} \mathrm{Cu}(111)$ reveal the pronounced interplay between the intermolecular interactions (including short-ranged steric effects, medium ranged van-der-Waals and longer ranged electrostatic interactions) and those between molecule and substrate. However, most importantly we have found that the 50 linear nature of the molecular arrays can be obtained for different stoichiometries and its lateral periodicity can thus be varied in a controlled way.

In conclusion, we have shown how control of the intermolecular interactions in binary supramolecular layers of 55 homeomorphic components can change the symmetry of their highly crystalline nanostructures. An exchange of fluorine and hydrogen atoms within a molecule can inhibit the formation of hydrogen bonds and change the structure of the binary layers from having a two- to a one-dimensional compositional ${ }_{60}$ periodicity. In addition, by changing the stoichiometry of the deposition we can tune the periodicity of the binary structures. This has been proved for molecular ratios between 2:1 and $1: 2$, but controllability of the periodicity over a larger range might be equally possible going to more disparate 
stoichiometries. Altogether, this letter reports on supramolecular self-assembly in which we obtain molecular templates with tunable symmetry and periodicity, a landmark in organic self-assembly of potential use for subsequent 5 synthesis of tailored functional molecular nanostructures.

Acknowledgement. D. G. O. acknowledges the Japanese Society for the Promotion of Science (JSPS) for a short-term postdoctoral fellowship.

\section{Notes and references}

$10{ }^{a}$ Donostia International Physics Center, Paseo Manuel Lardizabal 4, 20018 San Sebastián, Spain

${ }^{b}$ Advanced Electronic Materials Center, National Institute for Materials Science, 1-1 Namiki, Tsukuba 305-0044, Japan

${ }^{c}$ Max-Planck Institute for Metal Research, Heisenbergstr. 3, 70569

15 Stuttgart, Germany

${ }^{d}$ Institut de Ciencia de Materials de Barcelona, CSIC, Campus UAB, 08193 Bellaterra, Spain

${ }^{e}$ Deutsches Elektronen Synchrotron (DESY), Notkestr. 85, D-22607

Hamburg, Germany

$20{ }^{f}$ Centro de Física de Materiales (CSIC-UPV/EHU) - Materials Physics Center (MPC), Paseo Manuel Lardizabal 5, 20018 San Sebastián, Spain

${ }^{g}$ Departamento de Física Aplicada, Universidad del Pais Vasco, Pza. Oñate 2, 20018 San Sebastián, Spain.

* Current address: Lawrence Berkeley National Laboratory, Molecular

25 Foundry, 1 Cyclotron Road, Berkeley, CA 94720, USA. Tel: +1 510

4952074; E-mail: dgoteyza@lbl.gov

$\dagger$ Electronic Supplementary Information (ESI) available: Experimental details, larger scale STM images. See DOI: 10.1039/b000000x/

${ }^{1}$ H. L. Zhang, W. Chen, L. Chen, H. Huang, X. S. Wang, J. Yuhara, A. T. S. Wee, Small 2007, 3, 2015

${ }^{2}$ S. Berner, M. Corso, R. Widmer, O. Groening, R. Laskowski, P. Blaha, K. Schwarz, A. Goriachko, H. Over, S. Gsell, M. Schreck, H. Sachdev, T. Greber, J. Osterwalder, Angew. Chem. Int. Ed. 2007, 46, 5115

${ }^{3}$ N. Neel, J. Kröger, R. Berndt, Adv. Mater. 2006, 18, 174

${ }^{4}$ R. Otero, Y. Naitoh, F. Rosei, P. Jiang, P. Thostrup, A. Gourdon, E. Lægsgaard, I. Stensgaard, C. Joachim, F. Besenbacher, Angew. Chem. Int. Ed. 2004, 43, 2092

${ }^{5}$ F. Silly, A. Q. Shaw, K. Porfyrakis, G. A. D. Briggs, M. R. Castell, Appl. Phys. Lett. 2007, 91, 253109

${ }^{6}$ J. A. Theobald, N. S. Oxtoby, M. A. Phillips, N. R. Champness, P. H. Beton, Nature 2003, 424, 1029

${ }^{7}$ M. E. Cañas-Ventura, W. Xiao, D. Wasserfallen, K. Müllen, H. Brune, J. V. Barth, R. Fasel, Angew. Chem. Int. Ed. 2007, 46, 1814.

${ }^{8}$ M. Ruiz-Oses, N. Gonzalez-Lakunza, I. Silanes, A. Gourdon, A. Arnau, J. E. Ortega, J. Phys. Chem. B 2006, 110, 25573

${ }^{9}$ M. Treier, M.-T. Nguyen, N. V. Richardson, C. Pignedoli, D. Passerone, R. Fasel, Nano Lett. 2009, 9, 126

${ }^{10}$ S. De Feyter, F. C. De Schryver, Chem. Soc. Rev. 2003, 32, 139

${ }^{11}$ L. Grill, M. Dyer, L. Lafferentz, M. Persson, M. V. Peter, S. Hecht, Nat. Nanotech. 2007, 2, 687

${ }^{12}$ R. Gutzler, H. Walch, G. Eder, S. Kloft, W. M. Hecklab, M. Lackinger, Chem. Commun. 2009, 4456

13 A. Schiffrin, A. Riemann, W. Auwaerter, Y. Pennec, A. WeberBargioni, D. Cvetko, A. Cossaro, A. Morgante, J. V. Barth, Proc. Nat. Acad. Sci. 2007, 104, 5279

${ }^{14}$ A. Schiffrin, J. Reichert, W. Auwärter, G. Jahnz, Y. Pennec, A. WeberBargioni, V. S. Stepanyuk, L. Niebergall, P. Bruno, J. V. Barth, Phys. Rev. B 2008, 78, 035424.

15 Y. Pennec, W. Auwärter, A. Schiffrin, A. Weber-Bargioni, A. Riemann, J. V. Barth, Nat. Nanotech. 2007, 2, 99

${ }^{16}$ E. Barrena, D. G. de Oteyza, H. Dosch, Y. Wakayama, ChemPhysChem 2007, 8, 1915

${ }^{17}$ M. Ruiz-Oses, D. G. de Oteyza, I. Fernandez-Torrente, N. GonzalezLakunza, P. M. Schmidt-Weber, T. Kampen, K. Horn, A. Gourdon, A. Arnau, J. E. Ortega, ChemPhysChem 2009, 10, 896
${ }^{18}$ T. N. Krauss, E. Barrena, H. Dosch, Y. Wakayama, ChemPhysChem 2009, 10, 2445

${ }^{19}$ C. Bobisch, Th. Wagner, A. Bannani, R. Möller, J. Chem. Phys. 2003, 119, 9804

${ }^{20}$ W. Chen, H. Li, H. Huang, Y. Fu, H. L. Zhang, J. Ma, A. T. S. Wee, J. Am. Chem. Soc. 2008, 130, 12285

${ }^{21}$ K. J. Franke, G. Schulze, N. Henningsen, I. Fernandez-Torrente, J. I. Pascual, S. Zarwell, K. Rück-Braun, M. Cobian, N. Lorente, Phys. Rev. Lett. 2008, 100, 036807

${ }^{22}$ K. W. Hipps, L. Scudiero, D. E. Barlow, M. P. Cooke, J. Am. Chem. Soc. 2002, 124, 2126

${ }^{23}$ B. Xu, C. Tao, W. G. Cullen, J. E. Reutt-Robey, E. D. Williams, Nano Lett. 2005, 5, 2207

${ }^{24}$ L. Scudiero, K. W. Hipps, D. E. Barlow, J. Phys. Chem. B 2003, 107, 2903

${ }^{25}$ Y. Wakayama, D. G. de Oteyza, J. M. Garcia-Lastra, D. J. Mowbray, ACS Nano DOI: $10.1021 / \mathrm{nn} 102887 \mathrm{x}$

${ }^{26}$ F. Silly, U. K. Weber, A. Q. Shaw, V. M. Burlakov, M. R. Castell, G. A. D. Briggs, D. G. Pettifor, Phys. Rev. B 2008, 77, 201408

${ }^{27}$ P. Samori, N. Severin, C. D. Simpson, Klaus Mullen, J. P. Rabe, J. Am. Chem. Soc. 2002, 124, 9454

${ }^{28}$ M. de Wild, S. Berner, H. Suzuki, H. Yanagi, D. Schlettwein, S. Ivan, A. Baratoff, H.-J. Guentherodt, T. A. Jung, ChemPhysChem 2002, 10, 881

${ }^{29}$ B. Calmettes, S. Nagarajan, A.Gourdon, M. Abel, L. Porte, R. Coratger, Angew. Chem. Int. Ed. 2008, 47, 6994

${ }^{30}$ Y. L. Huang, W. Chen, H. Li, J. Ma, J. Pflaum, A. T. S. Wee, Small $2010,6,70$

${ }^{31}$ X. Sun, H. T. Jonkman, F.Silly, Nanotech. 2010, 21, 165602

${ }^{32}$ W. Chen, H. L. Zhang, H. Huang, L. Chen, A. T. S. Wee, Appl. Phys. Lett. 2008, 92, 193301

${ }^{33}$ N. Gonzalez-Lakunza, I. Fernandez-Torrente, K. J. Franke, N. Lorente, A. Arnau, J. I. Pascual, Phys. Rev. Lett. 2008, 100, 156805

${ }^{34}$ K. Suto, S. Yoshimoto, K. Itaya, J. Am. Chem. Soc. 2003, 125, 14976

${ }^{35}$ L. Chen, W. Chen, H. Huang, H. L. Zhang, J. Yuhara, A. T. S. Wee, Adv. Mater. 2008, 20, 484

${ }^{36}$ D. G. de Oteyza, J. M. García-Lastra, M. Corso, B. P. Doyle, L. Floreano, A. Morgante, Y. Wakayama, A. Rubio, J. E. Ortega, Adv. Funct. Mater. 2009, 19, 3567

${ }^{37}$ D. G. de Oteyza, I. Silanes, M. Ruiz-Oses, E. Barrena, B. P. Doyle, A.Arnau, H. Dosch, Y. Wakayama, J. E. Ortega, Adv. Funct. Mater. 2009, 19, 259

${ }^{38}$ D. E. Hooks, T. Fritz, M. D. Ward, Adv. Mater. 2001, 13, 227

${ }^{39}$ H. Huang, Y. Huang, J. Pflaum, A. T. S. Wee, W. Chen, Appl. Phys. Lett. 2009, 95, 263309

${ }^{40}$ H. Ishii, K. Sugiyama, E. Ito, K. Seki, Adv. Mater. 1999, 11, 605

${ }^{41}$ D. Vanderbilt, Surf. Sci. Lett. 1992, 268, L300

${ }^{42}$ K.-O. Ng, D. Vanderbilt, Phys. Rev. B 1995, 52, 2177

${ }^{43}$ D. G. de Oteyza, A. El-Sayed, J. M. Garcia-Lastra, E. Goiri, T. N. Krauss, A. Turak, E. Barrena, H. Dosch, J. Zegenhagen, A. Rubio, Y. Wakayama, J. E. Ortega, J. Chem. Phys. 2010, 133, 214703

${ }^{44}$ I. Chizhov, G. Scoles, A. Kahn, Langmuir 2000, 16, 4358

${ }^{45}$ D. G. de Oteyza, E. Barrena, H. Dosch, Y. Wakayama, Phys. Chem. Chem. Phys. 2009, 11, 8741

${ }^{46}$ D. G. de Oteyza, E. Barrena, M. Ruiz-Oses, I. Silanes, B. P. Doyle, J. E. Ortega, A. Arnau, H. Dosch, Y. Wakayama J. Phys. Chem. C 2008, 112, 7168

${ }^{47}$ H. Karakuban, M. Lange, J. Schaffert, O. Weingart, Th. Wagner, R. Möller, Surf. Sci. 2009, 603, L39

${ }^{48}$ The phase separated DIP regions do not show the same structure as on the bare $\mathrm{Cu}(111)$, but the structure observed for DIP on $\mathrm{Cu}(111)$ subject to confinement in terraces of widths below $15 \mathrm{~nm}$ [ref. 47]. The reason why this structure now extends to distances far above 15 $\mathrm{nm}$ remains unclear, but is unambiguously related to the presence of $\mathrm{CuPc}$. 